ISSN: $1679-3013$

D.O.I.: 10.5914/to. 2014.0108

\title{
CONDIÇÕES AMBIENTAIS DO ESTUÁRIO DO RIO TIMBÓ (PERNAMBUCO-BRASIL): BIOMASSA FITOPLANCTÔNICA E ALGUMAS VARIÁVEIS AMBIENTAIS
}

Recebido em: 19/09/2014
Aceito em: $20 / 10 / 2014$

\section{RESUMO}

O ambiente estuarino é altamente complexo e produtivo, além de ser um dos principais meios de subsistência para populações costeiras e ribeirinhas. Apesar de sua relevância ecológica, econômica e social, esse ecossistema vem sofrendo forte exploração antrópica, principalmente através da urbanização e do descarte de efluentes. Diante dessa situação, o presente estudo procurou avaliar a evolução das condições ambientais do estuário do rio Timbó, através da análise da clorofila a e das variáveis físico-químicas, comparando os resultados com trabalhos pretéritos. As coletas foram realizadas em quatro pontos fixos préestabelecidos, nos meses de setembro e novembro de 2013, representando os períodos chuvoso e estiagem respectivamente, na baixa-mar e preamar, durante a maré de sizígia. A pluviosidade e a maré influenciaram na maioria das variáveis
Fernando Antônio do Nascimento FEITOSA

Manuel de Jesus Flores MONTES

Danielle Caroline da Mota MELO

Jana Ribeiro de SANTANA

Laisa Madureira da SILVA

Simone Jorge FILHO

analisadas. A temperatura variou de $26.5^{\circ} \mathrm{C}$ a $29^{\circ} \mathrm{C}$, a salinidade entre 22 e 37 não sendo evidenciado estratificação na coluna d'água, a transparência oscilou de $0,5 \mathrm{~m}$ a $2,91 \mathrm{~m}$, o material particulado em suspensão foi de 6,4 a $17,0 \mathrm{mg} \cdot \mathrm{L}^{-1}$, a concentração do oxigênio dissolvido variou de 1,0 a 8,09 e sua taxa de $41,6 \%$ a $170,3 \%$. O teor de clorofila a variou de 2,16 a $37,99 \mathrm{mg} \cdot \mathrm{m}^{-3}$. As análises de clorofila a fracionada evidenciaram uma maior contribuição da fração menor que $20 \mu \mathrm{m}$ do fitoplâncton, onde a contribuição mínima foi de $57,07 \%$ e a máxima de $100 \%$. A clorofila a correlacionou-se positivamente com o oxigênio dissolvido e sua taxa de saturação e temperatura. As variáveis aqui analisadas indicaram que 0 ambiente encontra-se em processo de eutrofização como consequência do aumento da ação antrópica.

Palavras-chaves: Clorofila a; variáveis hidrológicas; qualidade ambiental;

\section{ABSTRACT}

The estuarine environment is highly complex and productive, besides being one of the main livelihoods for coastal and riverine populations. Despite its ecological economic and social relevance, is undergoing strong anthropic exploration, mainly through urbanization and effluents disposal. Therefore, the aim of this study was to evaluate the evolution of the environmental conditions of the estuary of the Timbó River, through the analysis of the chlorophyll $a$, and the physical and chemical variables, comparing the results with anterior works. The collections were made in four previously established fixed points, in the months of September and November 2013, representing the rainy season and dry season respectively, at low tide and high tide during the spring tide. Rainfall and tidal influenced most the analyzed variables. The temperature varied from $26.5^{\circ} \mathrm{C}$ to $29^{\circ} \mathrm{C}$, the salinity between 22 and 37 , and did not show stratification in the water column. The transparency oscillated from $0.5 \mathrm{~m} 2,91 \mathrm{~m}$, the suspended particles from 6.4 to $17,0 \mathrm{mg} \cdot \mathrm{L}^{-1}$, the concentration of dissolved oxygen ranging from 1.0 to $8.09 \mathrm{~d}$ and its rate $41.6 \%$ to $170.3 \%$. The chlorophyll $a$

1 - Universidade Federal de Pernambuco/Programa de Pós-Graduação em Oceanografia

2 - Departamento de Oceanografia/Universidade Federal de Pernambuco

Contatos: feitoc@bol.com.br; manuel@ufpe.br; melo.danielle@yahoo.com; jribeiro.pesca@gmail.com;

laisamadureira@hotmail.com; symonebiologa@hotmail.com. 
FEITOSA. F. A. do N. et al. Biomassa fitoplanctônica e variáveis hidrológicas do estuário do Rio Timbó (Pernambuco-Brasil).

analyses of the $<20 \mu \mathrm{m}$ fraction highlighted a higher contribution of this fraction of the phytoplankton, with contribution values ranging from $57 \%$ to $100 \%$. The chlorophyll a was positively correlated with dissolved oxygen, its saturation and temperature. The variables analyzed here indicated that the environment is in the process of eutrophication as a result of increased anthropogenic activities.

Keywords: Chlorophyll a; Hidrological variables; Environmental quality;

\section{INTRODUÇÃO}

Os estuários são caracterizados como corpos de águas costeiras, semi-fechados, e que apresentam livre conexão com o mar aberto, dentro dos quais a água do mar é gradativamente diluída pela água doce proveniente da drenagem terrestre (PRITCHARD, 1967; DAY JR. et al., 1989; ELLIOTT; MCLUSKY, 2002). Estes ambientes são altamente complexos e produtivos, especialmente devido às características hidrodinâmicas de circulação, que estimulam a produtividade primária local aprisionando nutrientes e organismos fitoplanctônicos (MIRANDA et al., 2002). Igualmente, atuam como área de alimentação, berçário, habitat, refúgio e reprodução para diversas espécies de importância ecológica e econômica, bem como consistem em um dos principais meios de subsistência para populações costeiras e ribeirinhas (BASTOS, FEITOSA e MUNIZ, 2005; FIGUEIREDO et al., 2007). Por apresentarem alto dinamismo e sofrerem constantes modificações (ESKINAZI-SANT'ANNA, TUNDISI, 1996), a caracterização hidrodinâmica de um estuário é fundamental para o entendimento dos processos geológicos, químicos, físicos e biológicos, que fornecem bases para conservação e uso sustentável destes ambientes (D'AQUINO et al., 2011).

Inserido neste contexto, o estuário do rio Timbó (Paulista, PE) é considerado como um dos corpos hídricos mais férteis do estado, apresentando altos índices de produtividade primária, e destacando-se como um dos principais contribuintes para a pesca artesanal de Pernambuco (COMPANHIA PERNAMBUCA DE RECURSOS HÍDRICOS, 2003). Parte considerável de sua bacia ainda conserva resquícios de Mata Atlântica, coqueirais, vegetação de praias, restingas, terraços litorâneos e manguezais, o que proporciona uma estrutura paisagística rica e diversificada ao estuário (BARBOSA, 2006).

Por décadas este ambiente vem sendo alvo dos mais diversos estudos (BARROS-FRANCA, PORTELA e MOURA, 1984; SILVA, 1989; SILVA-CUNHA, ESKINAZI-LEÇA e ALMEIDA, 1989; (KOENING, ESKINAZI-LEÇA, 1989; GREGO, 2004; GREGO et al., 2004), dentre os quais se pode destacar a avaliação realizada por (COSTA e MACÊDO, 1989), que diagnosticaram a área em como "não poluída", através da análise de parâmetros ambientais locais no período de 1984 a 1985.

Contudo, estudos mais recentes como os de FIGUEIREDO et al., (2007) e NORONHA, SILVA; e DUARTE (2010) demonstram que o mesmo ecossistema vem sofrendo forte exploração antrópica, com crescente movimento de ocupação e urbanização de populações ribeirinhas. O lançamento de efluentes domésticos e industriais cada vez mais frequente, deve estar contribuindo diretamente para a eutrofização deste corpo hídrico.

Desta forma, o presente trabalho teve como objetivo principal avaliar a evolução das condições ambientais do estuário do rio Timbó, fazendo uso deste relato como subsídio eficaz para futuro monitoramento da área.

\section{ÁREA ESTUDADA}

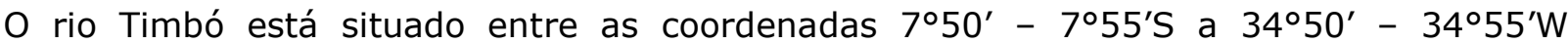
(FIDEM, 2003), no litoral de Pernambuco, entre os municípios de Abreu e Lima, Paulista e Igarassu, distante aproximadamente $30 \mathrm{Km}$ da capital, Recife (Fig. 01).

A bacia do rio Timbó é fortemente ocupada por residências e várias indústrias que contribuem com descarte de efluentes no corpo d’água, além da policultura e silvicultura às margens do rio (COMPANHIA PERNAMBUCANA RECURSOS HÍDRICOS, 2008). Essa região estuarina ocupa aproximadamente 1.397 ha e é constituída por duas paisagens. Na porção à montante, suas margens são marcadas por manguezal e subordinamente por coqueirais, incluindo também atividade industrial e descarte de efluentes. Na porção à jusante, a paisagem 
tem uma maior intervenção urbana através de casas, hotéis, restaurantes, marinas e piers, além de lançamento de efluentes domésticos (FIGUEIREDO et al., 2007). Apesar do uso indevido desse ambiente, o referido está inserido em uma Área de Proteção Ambiental (APA) desde 1986, segundo o decreto Lei No 9.931/86.

O litoral norte do estado de Pernambuco, localiza-se na área de domínio do clima tropical

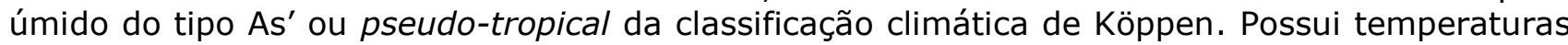
médias anuais que oscilam em torno dos $24^{\circ} \mathrm{C}$, nos meses de julho e agosto, e de $27^{\circ} \mathrm{C}$ em fevereiro, apresentando uma amplitude térmica de $3^{\circ} \mathrm{C}(\mathrm{CPRH}, 2003)$.

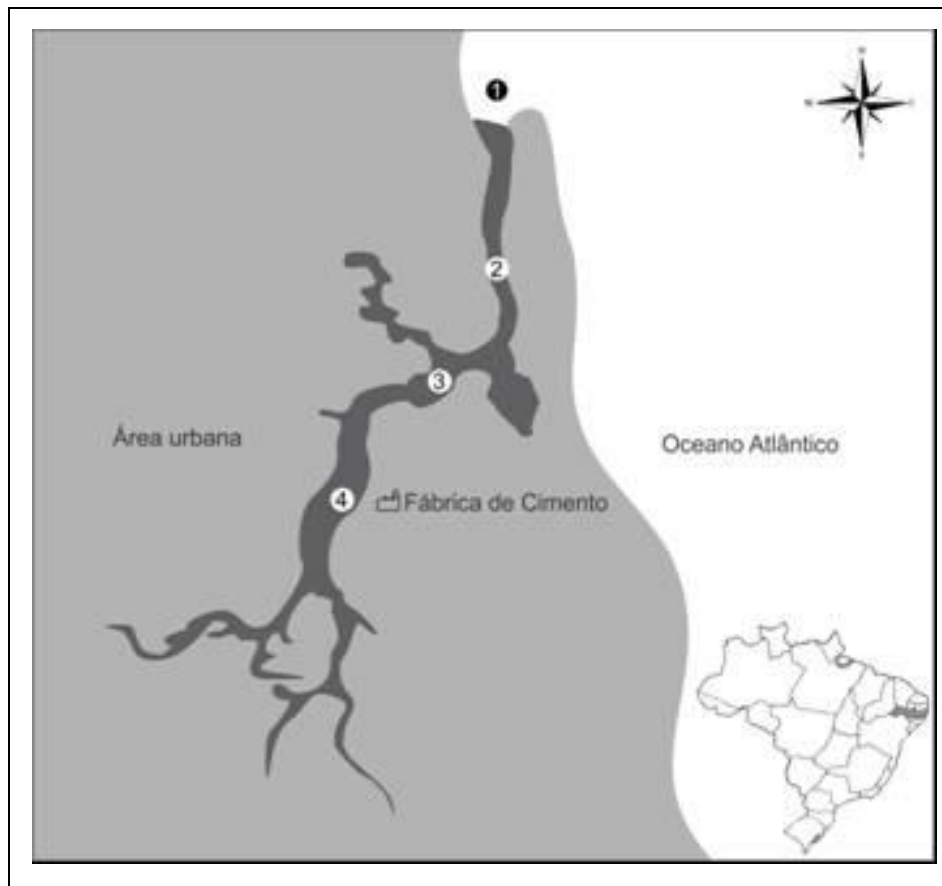

Figura 1 - Área estuarina do rio Timbó, no estado de Pernambuco, Brasil, onde estão localizados os pontos de coleta 1,2,3 e 4 (Fonte: Google Earth, 2014).

\section{MATERIAL E MÉTODO}

\section{Estações de coleta}

$\mathrm{Na}$ tabela a seguir estão discriminados as quatro estações de coleta na área amostral, de acordo com a porção do estuário do rio Timbó (Tabela 01).

Tabela 1 - Estações de coleta amostradas no estuário do rio Timbó (Paulista, PE).

\begin{tabular}{clcc}
\hline Estações & \multicolumn{1}{c}{ Estuário } & \multicolumn{1}{c}{ Lat (S) } & Long (W) \\
\hline 1 & Inferior (Foz) & $07^{\circ} 50^{\prime} 08.12^{\prime \prime}$ & $34^{\circ} 50^{\prime} 17.46^{\prime \prime}$ \\
2 & Médio (Jusante) & $0^{\circ} 51^{\prime} 26.20^{\prime \prime}$ & $34^{\circ} 50^{\prime} 27.80^{\prime \prime}$ \\
3 & Superior (Montante) & $0^{\circ} 52^{\prime} 15.25^{\prime \prime}$ & $34^{\circ} 51^{\prime} 14.39^{\prime \prime}$ \\
4 & Superior (Montante) & $07^{\circ} 52^{\prime} 41.08^{\prime \prime}$ & $34^{\circ} 52^{\prime} 41.08^{\prime \prime}$ \\
\hline
\end{tabular}

\section{Coleta dos dados}

As coletas foram realizadas nos meses de setembro representando o final do período chuvoso e novembro o de estiagem, na baixa-mar e preamar, durante a maré de sizígia.

As amostras de água para análise da clorofila a e hidrológica foram feitas na superfície utilizando uma garrafa Niskin de 3L. A Temperatura foi aferida in loco, através de um termômetro comum de álcool, a salinidade através de um refratômetro manual da Atago modelo S/Mill-E.

Os parâmetros como a profundidade e transparência da água foram aferidos com o auxílio do disco de Secchi. As concentrações de oxigênio dissolvido através da metodologia modificada de Winkler (STRICKLAND E PARSONS, 1972); a taxa de saturação do oxigênio pela tabela da 
United Nations Educational, Scientific and Culltural Organization (UNESCO, 1973). O material particulado em suspensão (MPS) através da metodologia de MELO et al. (1975). A biomassa fitoplanctônica (clorofila a) pelo método do espectrofotométrico da UNESCO (1966), descrito por Teixeira (1973). Para os cálculos utilizou-se a equação de PARSONS E STRICKLAND (1963), sendo os resultados expressos em $\mathrm{mg} \cdot \mathrm{m}^{-3}$.

Os dados de chuvas foram obtidos na estação meteorológica do Curado na cidade de Recife, distando cerca de $24 \mathrm{~km}$ da área estudada (INSTITUTO NACIONAL DE METEREOLOGIA, 2014).

\section{Análises estatísticas}

Para os testes de significância foi aplicado o teste-t após tentativa de normalização foi necessário realizar o teste Mann-Whitney, utilizando o programa computacional SigmaPlot versão 12.3. Para analisar a correlação entre as variáveis foi realizada a Análise de Componentes Principais (ACP) utilizando o programa computacional PRIMER 6.0 que realiza análises multivariadas.

\section{RESULTADOS}

\section{Pluviosidade}

O regime de chuvas no período estudado esteve dentro do padrão observado nos últimos 20 anos. Vale ressaltar que em setembro registrou-se um leve aumento na incidência pluviométrica, quando comparado com a média do mesmo mês num período de 20 anos. 0 total de chuvas para setembro/13 foi de $146.00 \mathrm{~mm}$, e de 76,00 mm para novembro/13 (Fig. 2).

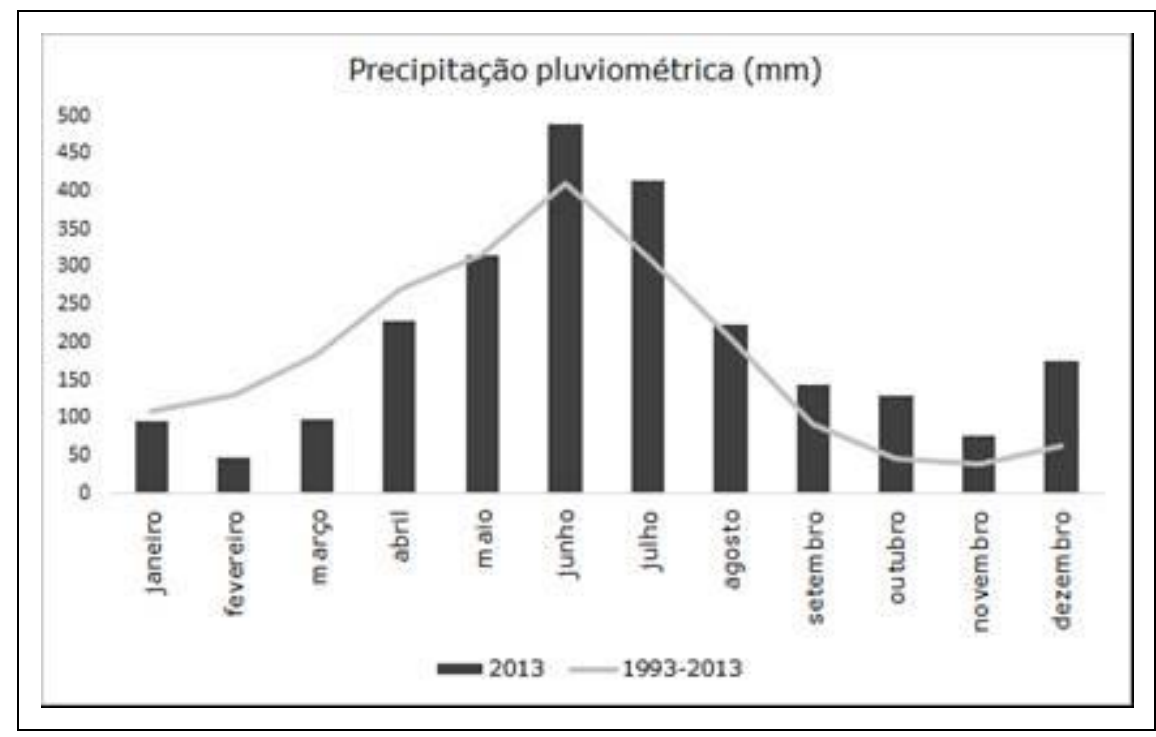

Figura 2 - Pluviosidade ( $\mathrm{mm}$ ), indicando a normal climatológica de chuvas correspondente aos últimos 20 anos (1993-2013) e referente aos meses de coleta (setembro e novembro de 2013).

\section{Temperatura}

A temperatura mais alta ocorreu na porção superior do estuário do rio Timbó, no mês de estiagem, registrando na baixa-mar (BM) e preamar (PM), $29^{\circ} \mathrm{C}$. Já o menor valor foi de $26,5^{\circ} \mathrm{C}$ obtido na porção inferior, no mês de setembro/13, durante a BM. A temperatura apresentou um valor médio de $27.7^{\circ} \mathrm{C}$. De acordo com o teste-t, houve diferença significativa em relação à sazonalidade, o mesmo não tendo sido observado para estágio de maré (Tabela 2).

\section{Salinidade}

As maiores taxas de salinidade foram verificadas na porção inferior do estuário, no mês de estiagem e na PM, com máxima de 37. Enquanto que a mínima de 22 ocorreu à montante, no 
FEITOSA. F. A. do N. et al. Biomassa fitoplanctônica e variáveis hidrológicas do estuário do Rio Timbó (Pernambuco-Brasil).

mês chuvoso, durante a BM; A média entre os meses foi de $29.5^{\circ} \mathrm{C}$. Após o uso do teste-t foi registrado variação significativa apenas para sazonalidade (Tabela 2 ).

\section{Profundidade Local}

As áreas mais profundas foram encontradas na foz do estuário, apresentando-se maior durante o mês chuvoso. As mais rasas foram observadas à montante. A profundidade média foi de 5,6m. Não houve variação significativa quanto à sazonalidade e maré (Tabela 2).

\section{Transparência da água}

Uma maior profundidade de transparência foi registrada em setembro com 1,85m. Enquanto que a menor foi registrada no mês chuvoso, devido a uma maior turbidez. O valor médio foi de $1,7 \mathrm{~m}$. Não foi verificada diferença significativa para a sazonalidade e maré (Tabela 2).

\section{Material Particulado em Suspensão}

O valor mais alto de MPS ocorreu na parte superior do estuário durante a PM $(17,00$ mg..$\left.^{1}\right)$; e o menor no mês de estiagem, no ponto 3, foi registrado o menor valor na PM $(3,7$ mg..$^{-1}$ ), sendo a média de $11,7 \mathrm{mg}^{-1}{ }^{-1}$. Após a aplicação do teste Mann-Whitney foi verificada diferença significativa somente em relação a estágio de maré (Tabela 2 ).

\section{Oxigênio dissolvido e Taxa de saturação do oxigênio}

A concentração mais elevada de oxigênio dissolvido na água foi observada na foz, no mês chuvoso, durante a PM $\left(8,09 \mathrm{ml}^{. \mathrm{I}^{-1}}\right)$. A mais baixa foi registrada na porção superior do estuário, no mês de estiagem, durante a PM $\left(1,00 \mathrm{ml}^{-1}{ }^{-1}\right)$. A maior taxa de saturação acompanhou a concentração do oxigênio, com o valor máximo na foz durante a PM (170,25\%), e o valor mínimo na porção superior do estuário durante a BM (41,59\%), ambos em setembro. Em relação ao oxigênio dissolvido foi verificado diferença significativa apenas para estágio de maré (Teste Mann-Whitney); O mesmo foi constatado para a taxa de saturação de oxigênio (Teste-t) (Tabela 2).

\section{Clorofila a}

Quanto à clorofila a as maiores concentrações foram registradas na foz, no mês chuvoso, durante a PM $\left(37,99 \mathrm{mg} \cdot \mathrm{m}^{-3}\right)$, e na parte superior do estuário no mês de estiagem, durante a BM $\left(32,92 \mathrm{mg} \cdot \mathrm{m}^{-3}\right)$. As menores concentrações foram $2,16 \mathrm{mg} \cdot \mathrm{m}^{-3}$ em BM e $3,16 \mathrm{mg} \cdot \mathrm{m}^{-3}$ em PM, ambas na foz, no mês de estiagem. Os resultados da clorofila fracionada muito se assemelharam aos da clorofila total, registrando maiores concentrações também na foz, no mês chuvoso, em PM $\left(27,95 \mathrm{mg} \cdot \mathrm{m}^{-3}\right)$ e na parte superior do estuário, no mês de estiagem, em BM $\left(27,87 \mathrm{mg} \cdot \mathrm{m}^{-3}\right)$. Já concentrações reduzidas ocorreram no mês de estiagem, com um valor de $2,34 \mathrm{mg} \cdot \mathrm{m}^{-3}$ no médio estuário, durante a BM; E 3,49 $\mathrm{mg} \cdot \mathrm{m}^{-3}$ na foz, durante a PM (Figura 03). Os valores médios da clorofila a total e fracionada foram de $20,0 \mathrm{mg} \cdot \mathrm{m}^{-3}$ e $15.1 \mathrm{mg} \cdot \mathrm{m}^{-3}$, respectivamente. Não houve variação significativa para sazonalidade e maré (Tabela 2 ). 
FEITOSA. F. A. do N. et al. Biomassa fitoplanctônica e variáveis hidrológicas do estuário do Rio Timbó (Pernambuco-Brasil).

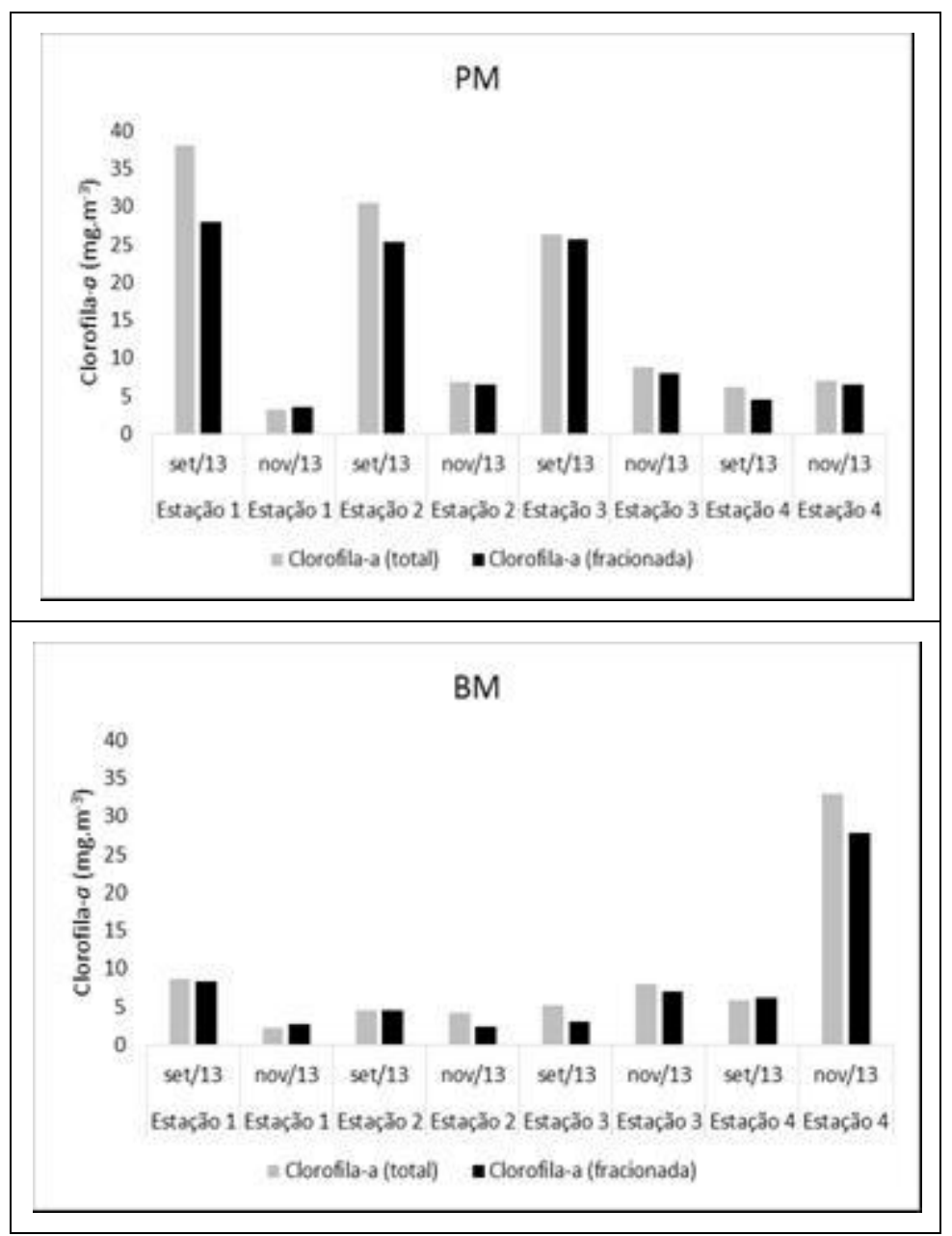

Figura 3 - Teores de clorofila a total e fracionada registradas nos meses de setembro e novembro de 2013, em ambos estágios de maré. 
Tabela 2 - Resultados dos parâmetros físicos-químicos encontrados no estuário do rio Timbó, registrando seus valores mínimo e máximo, durante a BM e a PM, nos meses de estiagem e chuvoso, nos quatro pontos de coleta.

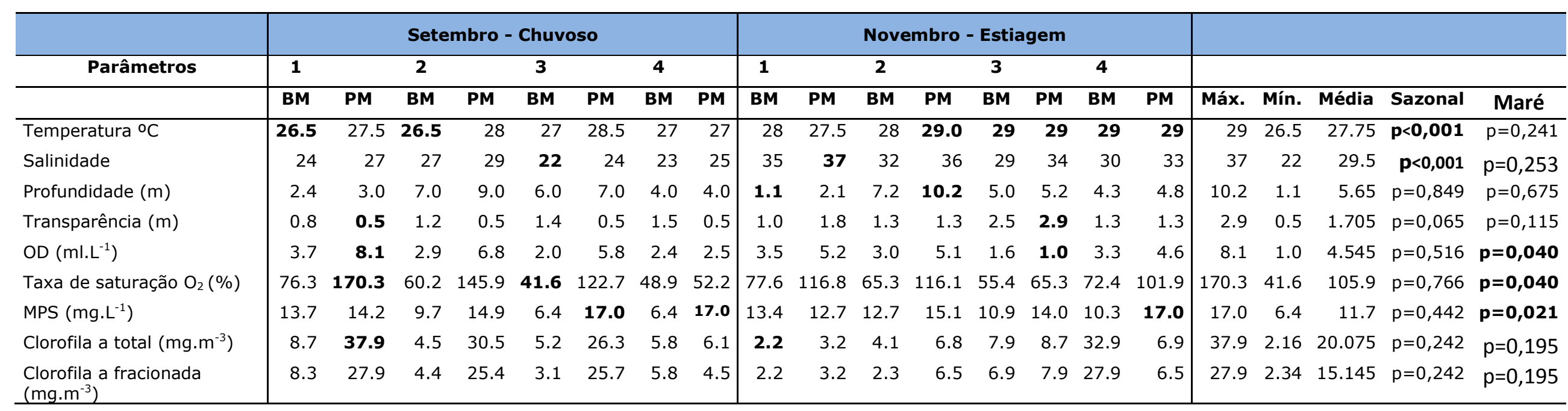

Tabela 3 - Variação dos dados e coeficientes de correlação entre as variáveis analisadas, das duas primeiras componentes da ACP, no estuário do rio Timbó, Brasil.

\begin{tabular}{lcc}
\hline & Componente/1 & Componente/2 \\
\hline \multicolumn{1}{c}{ Variáveis } & $(36,9 \%)$ & $(28,3 \%)$ \\
Clorofila-a (total) & $\mathbf{- 0 . 4 2 7}$ & -0.267 \\
Clorofila-a (fracionada) & $\mathbf{- 0 . 4 3 8}$ & -0.251 \\
O.D & $\mathbf{- 0 . 4 6 4}$ & 0.066 \\
Taxa. Satu.\% & $\mathbf{- 0 . 4 6 2}$ & 0.108 \\
Salinidade & -0.09 & $\mathbf{0 . 5 0 6}$ \\
Temperatura & $\mathbf{- 0 . 2 0 2}$ & 0.176 \\
Profundidade & -0.095 & $\mathbf{- 0 . 1 6 3}$ \\
Secchi & 0.323 & 0.164 \\
MPS & -0.109 & $\mathbf{0 . 5 1 2}$ \\
\hline
\end{tabular}

1 - Universidade Federal de Pernambuco/Programa de Pós-Graduação em Oceanografia

2 - Departamento de Oceanografia/Universidade Federal de Pernambuco

Contatos: feitoc@bol.com.br; manuel@ufpe.br; melo.danielle@yahoo.com; jribeiro.pesca@gmail.com; laisamadureira@hotmail.com;

symonebiologa@hotmail.com. 


\section{Análise das Componentes Principais (ACP)}

De acordo com a análise estatística foi possível observar que a primeira componente principal (PC1) explicou 36,9\% das variações e correlacionou positivamente clorofila a total, clorofila a fracionada e incersamente ao secchi. A segunda componente principal (PC2) explicou $28,3 \%$ das variações, correlacionando diretamente a salinidade e MPS, os quais apresentaramse inversos à clorofila a total e fracionada (Tabela 03).

\section{DISCUSSÃO}

Os ecossistemas tropicais são fortemente influenciados pela incidência das chuvas, apresentando-se de maneiras diferentes entre os períodos chuvoso e de estiagem (PASSAVANTE E FEITOSA, 2004). No estuário do rio Timbó, as principais forçantes físicas que alteraram as variáveis hidrológicas e as concentrações de clorofila a, foram a pluviosidade e a maré, o que foi igualmente observado por GREGO et al. (2005) para o rio Timbó; BASTOS et al. (2011) para a região estuarina do rio Maracaípe, e AQUINO et al. (2012) no rio dos Passos.

O presente estudo registrou temperaturas mais elevadas no mês de estiagem, mantendo uma relação inversa com a pluviosidade. Este padrão também foi descrito por trabalhos anteriormente desenvolvidos para a mesma área em questão (COSTA, MACÊDO, 1987/1989; SILVA, 1989; NORONHA; SILVA; DUARTE, 2010). Somado a este dado, um gradiente decrescente da temperatura foi observado em direção à jusante, podendo estar associado com a retenção da energia solar pelo solo lamoso como constatado anteriormente por GREGO et al. (2004).

Conforme observado pela análise de componentes principais a temperatura correlacionouse de forma positiva com a concentração e taxa de saturação do oxigênio e clorofila a, podendo ser consequência da maior interação entre o sedimento e a coluna d'água em estágio de preamar. De acordo com (SIPAÚBA-TAVARES, 1998 E PASSAVANTE E FEITOSA, 2004) a temperatura interfere na atividade biológica influenciando a distribuição e concentração do fitoplâncton no ambiente.

Em íntima associação com a temperatura, a salinidade é um dos parâmetros que mais influenciam uma comunidade biológica, pois como pode ser modificada em função da maré, acaba por se tornar uma variável determinante na distribuição dos organismos (FEITOSA et al., 1999). Para presente pesquisa não foi perceptível estratificação térmica nem salina indicando tratar-se de um estuário do tipo homogêneo.

Os dados de salinidade aqui apresentados demonstram uma variação sazonal, como consequência da pluviosidade como observado por NORONHA; SILVA; DUARTE (2010). O mesmo padrão foi encontrado por AQUINO et al. (2012), no estuário do rio dos Passos; Noriega et al. (2009) em Barra das Jangadas; HONORATO DA SILVA et al. (2009) no rio Formoso; e GREGO et al. (2009) para o estuário do rio Ariquindá.

O gradiente decrescente de salinidade observado em direção à montante é algo esperado para ecossistemas estuarinos, pois é na porção superior que o fluxo de água doce se faz mais evidente, corroborando trabalhos anteriores no estuário do rio Timbó (COSTA, MACÊDO, 1987/1989; SILVA, 1989, GREGO et al., 2004 e NORONHA, SILVA, DUARTE, 2010).

As áreas mais profundas das estabelecidas pelos pontos de coleta ocorreram durante a preamar em setembro. Este fato entra em acordo ao descrito por NORIEGA et al. (2009), cujo estudo demonstrou que a profundidade durante o período chuvoso foi mais que o dobro do que a registrada para o período de estiagem. AQUINO et al. (2012) no rio dos Passos, observaram que o ponto mais à jusante foi o mais profundo.

Normalmente, o estuário é caracterizado por ser uma região de pouca profundidade (COSTA et al., 2008) e este parâmetro está intimamente ligado com a topografia do sedimento, sofrendo interferência desta (BASTOS et al., 2011; AQUINO et al., 2012).

1 - Universidade Federal de Pernambuco/Programa de Pós-Graduação em Oceanografia

2 - Departamento de Oceanografia/Universidade Federal de Pernambuco

Contatos: feitoc@bol.com.br; manuel@ufpe.br; melo.danielle@yahoo.com; jribeiro.pesca@gmail.com;

laisamadureira@hotmail.com; symonebiologa@hotmail.com. 
FEITOSA. F. A. do N. et al. Biomassa fitoplanctônica e variáveis hidrológicas do estuário do Rio Timbó (Pernambuco-Brasil).

O estuário do rio Timbó, caracterizou-se como raso, esse fato pode estar associado ao forte processo de assoreamento provocado pela subtração da vegetação de mangue, para a construção de industrias, residências, hotéis, píeres, restaurantes, entre outros.

A transparência da água manifesta-se num gradiente crescente em direção à jusante (BRANCO et al., 2002), estando diretamente relacionada com a concentração de sais nutrientes e materiais particulados em suspensão (MOTA, 2003). Segundo AQUINO et al. (2012), a transparência da água é um parâmetro dependente da precipitação de chuvas e da topografia ambiente.

As maiores profundidades de transparência foram registradas no mês de estiagem, o que pode ser explicado pela redução das chuvas que alteram os índices de MPS. Tal padrão sazonal foi igualmente observado em trabalhos pretéritos para o estuário do rio Timbó (COSTA, MACÊDO, 1987/1989; GREGO et al., 2004, NORIEGA et al., 2009).

$\mathrm{Na}$ região superior do rio, a profundidade de transparência da água foi reduzida. Como citado anteriormente, a região encontra-se em um forte processo de assoreamento, o que pode ter contribuído fortemente para a redução desta variável. O mesmo foi observado por COSTA e MACÊDO (1987/1989); GREGO et al. (2004); FIGUEIREDO et al. (2007); e NORONHA, SILVA e DUARTE (2010) para o rio Timbó; e em outros estuários da região nordeste do Brasil (BRANCO, 2001; BRANCO et al., 2002; BASTOS, 2004; GREGO et al., 2009; AQUINO et al., 2012).

Vale ressaltar que no ponto de coleta mais interno, foi registrado uma intensa emissão de fuligem provinda de uma fábrica de cimento instalada às margens do rio, fator que pode ter alterado significativamente os valores de MPS e Secchi do local, sendo tal ocorrência também apontada por COSTA e MACÊDO (1987/1989). NORONHA, SILVA e DUARTE (2010), contudo, atribuíram a diminuição do Secchi ao aumento de matéria orgânica, provocados pelos constantes despejos de efluentes industriais na área.

O material particulado em suspensão é muito comum em regiões estuarinas, por apresentarem intenso hidrodinamismo e sofrerem influência de águas fluviais e oceânicas (BASTOS, 2002). O aumento do MPS diminui a transparência na coluna d'água, ocorrendo como um fator limitante para o desenvolvimento do fitoplâncton (CLOERN et al., 1985)

$\mathrm{Na}$ estação à jusante, onde o forte hidrodinamismo local, a constante movimentação de embarcações turísticas, atividade pesqueira, emissão de poluentes e a perda considerável de vegetações de mangue, podem ser citados como os principais fatores que levaram ao aumento do MPS. Na estação mais interna, este fato pode estar ligado às alterações na topografia local e à atividade industrial. Em seu estudo, GREGO et al. (2009) atribuíram o deságue do rio União, à elevada quantidade de MPS encontrada na baixa-mar, mais à montante do rio Ariquindá.

Quanto ao período, os maiores valores deste parâmetro ocorreram no mês chuvoso, o que pode ser explicado devido ao aumento no transporte de sedimento em sincronia com o movimento de vazão do rio; O contrário foi encontrado por GREGO et al., (2004), que registraram o pico de MPS no período de estiagem, durante a baixa mar.

A concentração e a taxa de oxigênio correlacionados a outros parâmetros ambientais são comumente usados para avaliar a qualidade de corpos hídricos (NORONHA; SILVA; DUARTE 2010; LIRA, 2008). Baixas taxas deste gás podem indicar poluição química, física ou biológica, enquanto que valores muito elevados estão associados ao processo de eutrofização (FLORESMONTES, 1996).

No presente estudo, os valores mais altos de oxigênio foram encontrados na foz durante a PM, quando ocorreu um maior fluxo de água. GREGO et al. (2004, 2009) e HONORATO DA SILVA et al. (2009), apontam como causas para este fato o maior aporte continental, e águas mais limpas e mais oxigenadas encontradas durante a PM, pelo efeito de circulação e mistura.

Valores mais elevados de oxigênio dissolvido e de sua saturação foram registrados durante o mês chuvoso, enquanto que valores reduzidos ocorreram durante o mês de estiagem. Diferente deste dado, NORIEGA et al. (2009), e GREGO et al. (2009) observaram maiores valores durante a estiagem, e menores durante o período chuvoso, não tendo sido encontrado 
uma variação sazonal. Igualmente a estes autores, NORONHA, SILVA e DUARTE (2010), também não relataram um padrão sazonal nem espacial para o rio Timbó.

Levando-se em consideração a classificação de MACÊDO e COSTA (1978), no presente trabalho, o estuário do rio Timbó apresentou-se variando de zona semi-poluída a supersaturada, prevalecendo a primeira. GREGO (2005) e FIGUEIREDO et al. já haviam alertado a perda da qualidade ambiental, tomando-se como base o teor de oxigênio dissolvido. O pico de valores altos de oxigênio encontrados à montante do rio Timbó é associado aos lançamentos constantes de efluentes (NORONHA, SILVA, DUARTE, 2010), que junto à ocupação urbana, são considerados as principais fontes poluidoras das águas do estuário (CPRH, 2000).

As concentrações da clorofila a é um parâmetro muito utilizado em estudos para caracterização da qualidade ambiental de corpos hídricos (CLOERN, JASSBY, 2010; GAMEIRO; BROTAS, 2010). Diversos agentes externos, sejam antropogênicos ou naturais, interferem na biomassa fitoplanctônica, sendo a precipitação de chuvas considerada o mais relevante dentre eles (PASSAVANTE e FEITOSA, 2004).

As maiores concentrações de clorofila a corresponderam ao mês chuvoso, e as menores ao de estiagem possivelmente em resposta ao aumento dos nutrientes inorgânicos, o que deve ter estimulado o crescimento da biomassa algal .Esta variação sazonal também foi constatada por outros autores, em estudos nestes e em outros estuários (MOURA, 1991, BRANCO, 2001, BASTOS, 2002, HONORATO DA SILVA, 2003, GREGO et al., 2004; SANTIAGO et al., 2005; HONORATO DA SILVA et al., 2009).

Ainda se pode destacar que no mês de estiagem, na estação mais à montante e no estágio de maré baixa, foi registrado um pico no teor de clorofila $a$, onde o efeito dos efluentes é mais evidente, o que pode ter contribuído com a elevação da biomassa fitoplanctônica local. A ocorrência de uma maior concentração de clorofila a no período de estiagem é característica de ambientes que sofrem com intermitentes ações antrópicas (GREGO et al., 2004; FEITOSA, 1988; FEITOSA et al., 1999).

Valores mais reduzidos de concentrações de clorofila a foram registradas na foz, durante a estiagem. Isto pode ser explicado em virtude da maior diluição de nutrientes quando estes chegam à foz, fato que foi igualmente apontado por GREGO et al. (2004).

Quando comparado a atual situação do estuário do rio Timbó com trabalhos anteriormente desenvolvidos (GREGO et al., 2005; FIGUEIREDO et al., 2007), verifica-se que as concentrações de clorofila a continuam muito elevadas, o que coincide com o desenvolvimento cada vez mais evidente da área urbana.

\section{CONCLUSÕES}

As variáveis ambientais oscilaram em função da pluviosidade e da altura da maré. O ambiente caracterizou-se com raso, e não estratificado, com presença de bancos de areia ao longo de toda zona estuarina.

Os altos teores de clorofila a e a taxa de saturação do oxigênio foram indicativos de um ambiente fortemente impactado. Na década de 80, o estuário do rio Timbó foi classificado apenas como uma zona supersaturada e de baixa saturação; atualmente, é considerado como uma área semi-poluída, principalmente em sua montante, onde é observado maior aporte continental e menor contribuição marinha.

O lançamento de efluentes domésticos e industriais está aumentando, o que tende a acelerar o processo de eutrofização, com um gradativo declínio ambiental nessa região.

\section{AGRADECIMENTOS}

Os autores desejam agradecer ao Programa de Pós-Graduação em Oceanografia (PPGO/UFPE), pelo financiamento das campanhas que viabilizaram a realização deste estudo e aos Laboratórios de Química (LOQUIM) e do Fitoplâncton (LABFITO), pela realização das análises indispensáveis à elaboração e interpretação dos resultados aqui apresentados. 
FEITOSA. F. A. do N. et al. Biomassa fitoplanctônica e variáveis hidrológicas do estuário do Rio Timbó (Pernambuco-Brasil).

\section{REFERÊNCIAS BIBLIOGRÁFICAS}

AQUINO, E. P et al. Biomassa fitoplanctônica e fatores ambientais em um estuário tropical do Brasil. Tropical Oceanography. Recife, v. 40, n.1. p. 17-28, 2012.

BARROS-FRANCA, L. M.; PORTELA, O. C.; MOURA, R. T. Aspectos prelimires do microfitoplâncton na zona interior do estuário do Rio Timbó e zona adjacente (Paulista, PE). Cadernos Ômega, Recife, v. 1, n.1, p. 17-27, 1984.

BRANCO, E. S.; FEITOSA, F. A. N.; FLORES-MONTE, M. J. Variação sazonal e espacial da biomassa fitoplanctônica relacionada com parâmetros hidrológicos no estuário de Barra das Jangadas (Jaboatão dos Guararapes - Pernambuco - Brasil). Tropical Oceanography, Recife, v. 30, n. 2, p. 79-96, 2002.

BASTOS, R. B. Variação espaço-temporal da biomassa fitoplanctônica relacionada com parâmetros abióticos no estuário do rio Una (Pernambuco-Brasil). 2002, 56 f. Monografia (Graduação em Ciências Biológicas). Universidade Federal de Pernambuco, 2002.

BASTOS, R. B. et al. Caracterização de uma zona costeira tropical (Ipojuca, Pernambuco Brasil): produtividade fitoplanctônica e outras variáveis ambientais. Brazilian Journal of Aquatic Science and Technology, Florianópolis, v. 15, n. 1, p. 01-10, 2011.

BASTOS, R. F.; FEITOSA, F. A. N.; MUNIZ, K. Variabilidade espaço-temporal da biomassa fitoplanctônica e hidrologia no estuário do rio Uma (Pernambuco-Brasil). Tropical Oceanography, Recife, v.33, n. 1, p. 1-18, abr/dez. 2005.

BARBOSA, M. J. O. Estudos sedimentológicos do estuário do rio Timbó, PE. Recife: 2006, 93f. Dissertação (Mestrado em Geociências) - Centro de Tecnologia e Geociências. Universidade Federal de Pernambuco. 2006.

CLOERN, J. E. et al. Temporal dynamics of estuarine phytoplankton - a case-study of SanFrancisco Bay. Hydrobiologia, v. 129, p. 153-176, 1985.

CLOERN, J. E.; JASSBY, A. D. Patterns and Scales of Phytoplankton Variability in EstuarineCoastal Ecosystems. Estuaries and Coasts, EUA, v. 33, p. 230-241, 2010.

CPRH 2003. Recursos Hídricos Superficiais. Disponível em: http:// www.cprh.pe.gov.br/, acessado em 05/09/2014.

$\mathrm{CPRH}, 2008$. Recursos Hídricos Superficiais. Disponível em: http:// www.cprh.pe.gov.br/, acessado em 05/09/2014.

COSTA, K. M. P., MACÊDO, S. J. Estudo Hidrológico do Rio Timbó. Trabalhos Oceanográficos, Recife, v. 20, p.7-34, 1989.

Costa K. M. P. Hidrologia e Biomassa Primária. Recife: 1991, 217f. Tese (Doutorado em Oceanografia) - Centro de Tecnologia e Geociências. Universidade Federal de Pernambuco. 1991.

COSTA, K. M. P.; MACÊDO, S. J. Estudo hidrológico do rio Timbó. Trabalhos Oceanográficos, Recife, v.20, p. 7-34, 1987/89.

DAY JR, J. W. et al. Estuarine Ecology, New Jersey: Wiley, p.558, 1989.

D'AQUINO, C. A. et al. F. Caracterização oceanográfica e do transporte de sedimentos em suspensão no estuário do Rio Mampituba, SC. Rev. Bras. Geof., São Paulo, v. 29, n. 2, p. 217230, 2011.

DAMME, V. D. et al. Spatialand temporal pattern sofwater quality along the estuarine salinity gradient of the Scheldt estuary (Belgiumand The Netherlands): results of an integrated monitoring approach. Hydrobiologia, v. 540, p. 29-45, 2005.

EDWARDS V. R.; TETT, P.; JONES K. J. Changes in the yield of chlorophyll a from dissolved available inorganic nitrogen after an enrichment event applications for predicting eutrophication in coastal waters. Continental Shelf Research. p. 1-15. 2003. 
FEITOSA. F. A. do N. et al. Biomassa fitoplanctônica e variáveis hidrológicas do estuário do Rio Timbó (Pernambuco-Brasil).

ELLIOTT, M., MCLUSKY, D. S. 2002. The need for definitions in understanding estuaries. Estuarine, Coastal and Shelf Science, v. 55, n. 6, p. 815-827, nov/fev. 2002.

ESKINAZI-SANT'ANNA, E. M.; TUNDIS, J. G. Zooplâncton do estuário do Pina (RecifePernambuco-Brasil): composição e distribuição temporal. Revista Brasileira de Oceanografia, São Paulo, v. 44, n. 1, p. 23-33, 1996.

FEITOSA, F. A. N.; NASCIMENTO, F. C. R.; COSTA, K. M. P. Distribuição espacial e temporal da biomassa fitoplanctônica relacionada com parâmetros hidrológicos na Bacia do Pina (Recife, PE). Trabalhos Oceanográficos, Recife, v. 27, n. 2, p. 1 - 13, 1999.

FIDEM. Fundação de Desenvolvimento da Região Metropolitana do Recife, CONDEPE. Tamandaré: um arrasto de artes e oficinas. CONDEPE, 44p. 2003.

FIGUEIREDO, J. A. et al. Evolução físico-química de águas do estuário do rio Timbó: um caso de reavaliação ambiental (1984-2003). Estudos Geol., Recife, v. 17, n.1, p. 85-104, 2007.

FLORES MONTES, M. J. Variação nictemeral do fitoplâncton e parâmetros hidrológicos no canal de Santa Cruz, Itamaracá, PE. Recife, PE: 1986. 197f. Dissertação (Mestrado em Oceanografia Biológica) - Centro de Tecnologia e Geociências, Universidade Federal de Pernambuco. 1996.

GAMEIRO, C.; ZWOLINSKI, J.; BROTAS, V. Light control on phytoplankton production in a shallow and turbid estuarine system. Hydrobiologia, v. 669, p. 249-263, 2011.

GRASSHOF, K.; EHRHARDT, M.; KREMLING, K. Methods of seawater analysis, New York, v. 2, 419p., 1983.

GREGO, C. K. S. Distribuição especial e sazonal da composição e biomassa fitoplanctônica correlacionada com a hidrologia do estuário do rio Timbó (Paulista, Pernambuco, Brasil). Recife, PE: 2004. 116f. Dissertação (Mestrado em Oceanografia Biológica), - Centro de Tecnologia e Geociências, Universidade Federal de Pernambuco. 2004.

GREGO, C. K. S. et al. Distribuição espacial e sazonal da clorofila a fitoplanctônica e hidrologia do estuário do rio Timbó (Paulista, PE). Tropical Oceanography, Recife, v. 32, n. 2, p. 181199, 2004.

GREGO, C. K. S. et al. Fitoplâncton do ecossistema estuarino do rio Ariquindá (Tamandaré, Pernambuco, Brasil): variáveis ambientais, biomassa e produtividade primária. Atlântica, Porto Alegre, v. 31, n. 2, p. 183-198, 2009.

HONORATO DA SILVA, M. et al. Estrutura sazonal e espacial do microfitoplâncton no estuário tropical do rio Formoso, PE, Brasil. Acta bot. Bras., v. 23, n. 2, p. 355-368, 2009.

INMET, 2014. Índice de chuvas da cidade do Recife. Disponível em:http://www.inmet.gov.br/portal/. Acessado em: 01/09/2014.

KOENING, M. L.; ESKINAZI-LEÇA, E. Biomassa e fracionamento do fitoplâncton no estuário do rio Timbó. Trabalhos Oceanográficos, Recife, v. 20, p. 53-76, 1989.

LIRA, J. B. de M. Avaliação preliminar das concentrações de metais pesados nos sedimentos da Lagoa do Araçá, Recife- Pernambuco, Brasil. 2008, 77 f. Dissertação (Mestrado em Tecnologia Ambiental) - Instituto de Tecnologia de Pernambuco (ITEP). 2008.

LOSADA, A. P. M.; FEITOSA, F. A. N.;LINS, I. C. Variação sazonal e espacial da biomassa fitoplanctônica nos estuários dos rios Ilhetas e Mamucaba (Tamandaré-PE) relacionada com parâmetros hidrológicos. Tropical Oceanography, Recife, v. 31, n. 1, p. 1-26, 2003.

MACÊDO, S. J.; COSTA, K. M. P. Estudo ecológico da região de Itamaracá, Pernambuco-Brasil, condições hidrológicas do estuário do rio Botafogo. Ciência e Cultura, São Paulo, v. 30, n. 7, 1978.

MELO, U.; SUMMERHAYERS, C. P.; TONER, L. G. Metodologia para o estudo do material em suspensão na água do mar. Boletim Técnico da Petrobrás, Rio de Janeiro, v.18, n. 3/4, p.115-127, 1975. 
FEITOSA. F. A. do N. et al. Biomassa fitoplanctônica e variáveis hidrológicas do estuário do Rio Timbó (Pernambuco-Brasil).

MIRANDA, L. B., CASTRO, B. M., KJERFVE, B. Princípios de Oceanografia física de estuários. São Paulo, EDUSO, 2002, 414 p.

MOREIRA, M.O.P. Produção do fitoplâncton em um ecossistema estuarino tropical. (Estuário do rio Cocó, Fortaleza, Ceará). Recife, 1994, 338 f. Dissertação (Mestrado em Oceanografia Biológica) - Centro de Tecnologia e Geociências, Universidade Federal de Pernambuco. 1994.

MOURA, R. T. Biomassa, produção primária do fitoplâncton e alguns fatores ambientais na Baía de Tamandaré. Rio Formoso. Pernambuco. Brasil. Recife, PE: 1991. $209 f$. Dissertação (Mestrado em Oceanografia). Departamento de Oceanografia, Universidade Federal de Pernambuco, 1991.

MOURA, R. T. Biomassa, produção primária do fitoplâncton e alguns fatores ambientais na Baía de Tamandaré. Rio Formoso. Pernambuco. Brasil. Recife, PE: 1991. $209 f$. Dissertação (Mestrado em Oceanografia) - Centro de Tecnologia e Geociências, Universidade Federal de Pernambuco. 1991.

NORIEGA, C. A. et al. Series temporales de variables hidrobiológicas em um estuário tropical (Brasil). Revista de Biología Marina y Oceanografía, Chile, v. 44, n. 1, p. 93-108, abr/2009.

NORONHA, T. J. M.; SILVA, H. K. P.; DUARTE, M. M. M. B. Avaliação dos impactos antrópicos e a qualidade da água do Estuário do Rio Timbó, Pernambuco, Brasil. Revista de Ciência, Tecnologia e Humanidades do IFPE, Recife, v. 2, n. 1, fev/2010.

PARSONS, T. R.; STRICKLAND, J. D. H. Discussion of spectrophotometric determination of marine plankton pigments, with revised equations of as certaining clorhophyll a and caratenoids. Journal of Marine Research, New Haven, v.21, n. 3, p.155-163, 1963.

PASSAVANTE, J. Z. O. Produção primária do fitoplâncton do Canal de Santa Cruz (Itamaracá, Pernambuco). São Paulo: 1979, 188f. Tese (Doutorado em Oceanografia Biológica) - Instituto Oceanográfico, Universidade de São Paulo. 1979.

PASSAVANTE, J. Z. O.; FEITOSA, F. A. N. Dinâmica da produtividade fitoplanctônica na zona costeira marinha. In: Eskinazi-Leça, E.; Newmann-Leitão, S.; Costa, M. F. Oceanografia: um cenário tropical. Recife: Bagaço, 2004. 761 p.

PRITCHARD, D. W. What is an estuary, Physical View Point. In: Lauf, G. H. (Ed.) Estuaries, American Association for the Advancement of Science. Washington D.C., n. 83, p. 3-5, 1967.

SANTOS-FERNANDES T. L. et al. Fitoplâncton do estuário do Rio Jaguaribe (Itamaracá, Pernambuco, Brasil): Biomassa. Trabalhos Oceanográficos, Recife, v. 26, n. 2, p.1-18, 1998.

SILVA, J. V. Produção Primária do Fitoplâncton do Estuário do Rio Timbó (Paulista-PE). Recife, PE: 1989. 83 f. Dissertação (Mestrado em Criptógamos) - Centro de Ciências Biológicas, Universidade Federal de Pernambuco, 1989.

SILVA-CUNHA, M. G. G.; ESKINAZI-LEÇA, E.; ALMEIDA, C. D. P. Taxonomia e Ecologia do microfitoplâncton do estuário do rio Timbó. Trabalhos Oceanográficos, Recife, v. 20, p. 3552, 1989.

SIPAÚBA-TAVARES, L. H. Limnologia dos sistemas de cultivos. In: VALENTI, W. C, (Ed.), Carcinicultura de Água Doce: Tecnologia para Produção de Camarões. Brasília, IBAMA/FAPESP, Cap. 3, p. 47-75, 1998.

STRICKLAND, J. D. H., PARSONS, T. R. A. Pratical handbook of seawater analysis. Bulletin Fisheries Research Board of Canada, Ottawa, n. 167, p. 207-211, 1972.

TEIXEIRA, C. Introdução aos métodos para medir a produção primária do fitoplâncton marinho. Boletim do Instituto Oceanográfico da Universidade de São Paulo, v. 22, p. 59-92, 1973.

TORRES, F. T. P.; MACHADO, P. J. O. Introdução à climatologia. São Paulo: Cengage Learning. 2011. 280p. 
FEITOSA. F. A. do N. et al. Biomassa fitoplanctônica e variáveis hidrológicas do estuário do Rio Timbó (Pernambuco-Brasil).

UNESCO. Determination of photosynthethic pigments in sea water. Report of SCOR/UNESCO, working group 17 meat from 4 to 6 june, 1964, Paris. 69p. (Monographys on Oceanography Methodology, 1). 1966.

UNESCO. International Oceanographic Table, Wormy, n. 2, 141p. 1973. 